\title{
IDENTIFICATION AND PREDICTION OF USER BEHAVIOR DEPENDING ON THE CONTEXT OF THE USE OF SMART MOBILE DEVICES
}

\author{
Siniša Husnjak, Dragan Peraković, Ivan Forenbacher, Ivan Jovović \\ University of Zagreb, Faculty of Transport and Traffic Sciences, Vukelićeva 4, 10000 Zagreb, Republic of Croatia
}

\begin{abstract}
Smart mobile devices (smartphones) represent an important factor in access to information and communications networks. The wide population of users with different demands is using smart mobile devices in many contexts. The context of use of smart mobile devices represents a joint impact of smart mobile devices and mobile applications, used information and communications services and the user's environment. The integration of smart mobile devices into user's everyday life is the background for the development of the conceptual model that will make conclusions about their habits and patterns of their behavior based on the collected data. The data analysis can determine user's affiliation to a particular segment of users. This paper will analyze new system for measurement, data analysis and reporting of the use of smart mobile devices and related information and communication services, depending on the context of use.
\end{abstract}

Keywords: Smart mobile devices; Context; User behavior; Segmentation; Behavior patterns
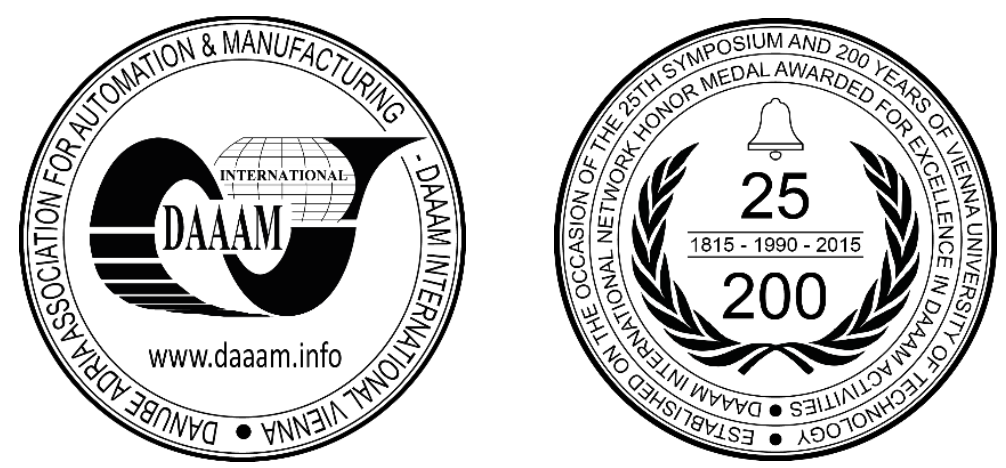

This Publication has to be referred as: Husnjak, S[inisa]; Perakovic, D[ragan]; Forenbacher, I[van] \& Jovovic, I[van] (2016). Identification and Prediction of User Behavior Depending on the Context of the Use of Smart Mobile Devices, Proceedings of the 26th DAAAM International Symposium, pp.0462-0469, B. Katalinic (Ed.), Published by DAAAM International, ISBN 978-3-902734-07-5, ISSN 1726-9679, Vienna, Austria

DOI: $10.2507 / 26$ th.daaam.proceedings.061 


\section{Introduction}

Smartphones are an indispensable part of modern communication and people spend considerable time interacting with their devices every day [1]. The popularity of smartphones is increasing among people all over the world [2]. The number of people adopting mobile phones is surging globally, and accessing the Internet using advanced mobile handsets and mobile usage of laptop computers is becoming more common [3].

By the end of 2014, the number of mobile-connected devices exceeded the number of people on earth, and by 2019 there will be nearly 1.5 mobile devices per capita. Globally, 54 percent of mobile devices will be smart devices by 2019, up from 26 percent in 2014. Smart mobile devices refers to mobile connections that have advanced multimedia/computing capabilities with a minimum of $3 \mathrm{G}$ connectivity [4]. By 2016 the number of smartphone subscriptions will exceed those for basic phones as they become increasingly affordable in developing markets. One of the main reasons for the rapid growth in smartphone subscriptions is that subscribers in Asia Pacific, the Middle East and Africa are exchanging their basic phones for smartphones [5].

As smartphones provide more and more applications for an increasingly wider range of usage situations, they become an increasingly integrated part of user's everyday life. The programmability of smartphones gives a possibility to turn the devices into data collection platforms providing detailed information on user's smartphone usage habits [6]. The widespread dissemination of smartphones has enabled pervasive sensing environments that allow collecting and capturing the user's context, which refers to information that can help characterize a user's activity or status in a given situation [7].

Mobile usage situations vary a lot in terms of the technical, physical and social context of use. Thus the services cannot be designed only for specific contexts of use but need to be adapted to different users and usage situations. Together, personalisation and context-awareness can greatly improve the usability of mobile services by providing the users with contextually and personally relevant information and functions. A major design challenge is to predict the personal needs in varying contexts of use without requiring constant attention from the user in the form of personalisation dialogues and refining questions [8].

A significant number of smartphone users includes very large number of users with different user's characteristics. Considering the fact that users carry their smartphone devices with them daily, smartphone devices allow collecting the data about their owners. Considering the potential of monitoring users of the smartphone devices, the number of smartphone sensors and the amount of user's data, smart mobile devices present an excellent platform for collecting data about their owners.

The aim of the research and a research question is how to produce a conceptual model which will allow identification and segmentation of smartphone users. The presented model in the research provides the technical solution for the possibility of collecting and analysis of the user's data. Collected information data and its subsequent analysis allow identification and segmentation of different groups of users according to their behavior and habits.

\section{Usage of smart mobile devices}

Smart mobile devices are multi-functional terminal devices with options such as: Internet access, using of applications, e-mail communication, messaging, data storage and using of multimedia files [9]. Average smartphone usage grew 45 percent in 2014. Smartphones represented only 29 percent of total global handsets in use in 2014, but represented 69 percent of total global handset traffic [4]. Today's smartphones have a touch screen that allows users intuitive usage of their mobile devices. Users have their smartphones always with them and they are often used as a substitute for GPS navigation, digital camera or video camera, media player, etc [10].

In general, younger consumers around the globe are more likely to embrace smartphone devices. It can be expected that their interest will help drive smartphone adoption as they age and make up a greater percentage of the consumer base [11].

\subsection{Smart mobile devices penetration growth}

Mobile subscribers are growing rapidly, and bandwidth demand due to data and video is increasing [1]. The number of mobile data subscriptions is increasing rapidly. Around the world, smartphone uptake has continued at a strong pace. 65-70 percent of all mobile phones sold in Q3 2014 were smartphones [5].

The number and variety of devices on the mobile network continue to increase. By 2019, there will be 8.2 billion handheld or personal mobile-ready devices. Regionally, North America and Western Europe are going to have the fastest growth in mobile devices and connections with 22 percent and 14 percent CAGR (Compound Annual Growth Rate) from 2014 to 2019, respectively. A rapid decline is seen in the share of non-smartphones from more than 61 percent in 2014 (4.5 billion) to less than 27 percent by 2019 (3.1 billion). The share of smart devices and connections as a percentage of the total will increase from 26 percent in 2014 to more than half, at 54 percent, by 2019, growing 3.3 -fold during the forecast period (Figure 1) [4]. 


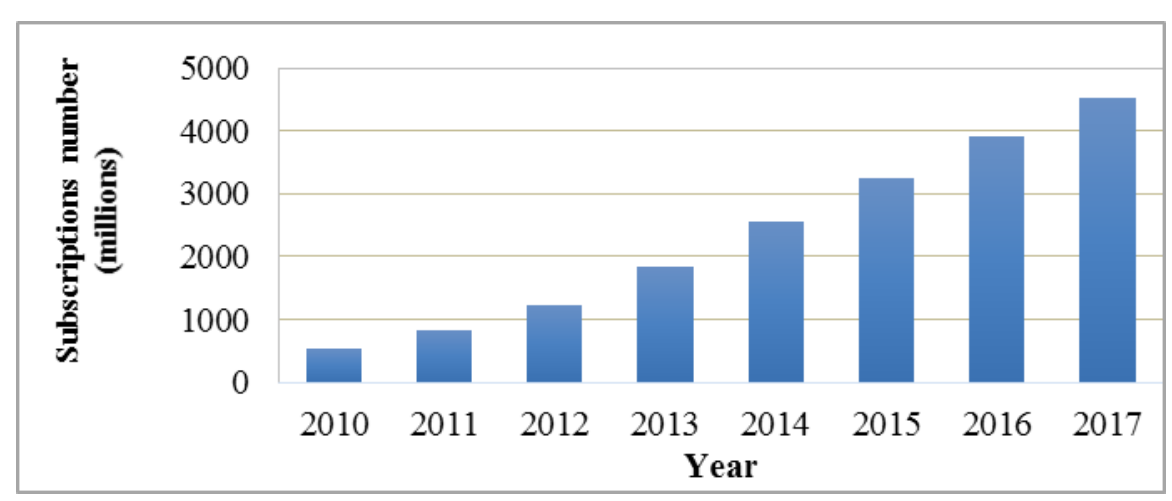

Fig. 1. Global smartphone subscriptions growth, 2010 - 2017, [12]

With mobile phone penetration at or above $100 \%$ in many markets worldwide, growth in the mobile industry relies now more than ever on existing subscribers upgrading to new devices and technologies and using new applications and services [13]. Because of increased usage on smartphones, smartphones will reach three-quarters of mobile data traffic by 2019. By the end of 2019 North America will have nearly 90 percent of its installed base converted to smart devices and connections, followed by Western Europe with 78 percent smart devices and connections [4].

\subsection{Smart mobile devices data traffic usage}

The continuous increase in the average amount of smartphone data traffic per one subscriber contract globally leads to an increase in the total mobile data traffic.

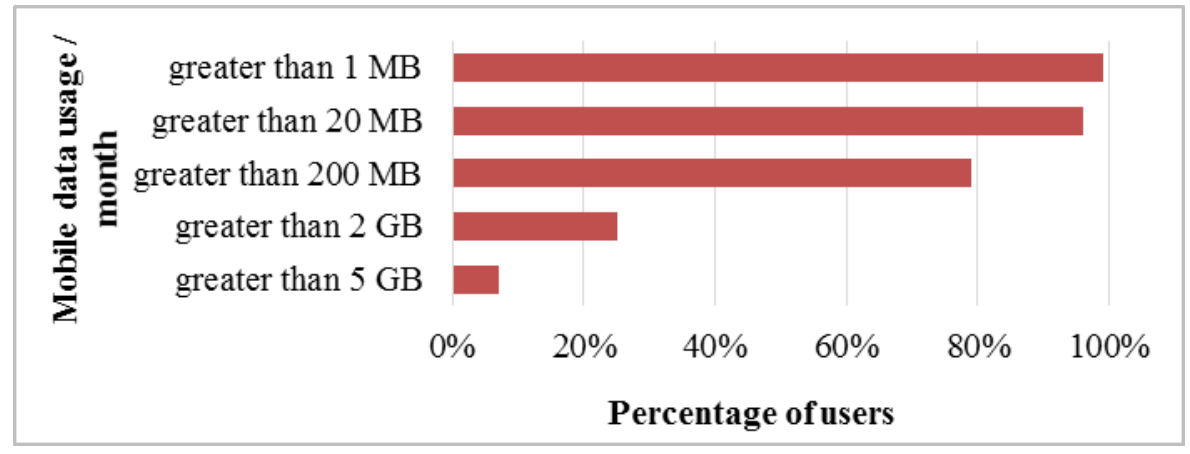

Fig. 2. Percentage of users and their mobile data traffic usage, [14]

While mobile phones were once used primarily for voice communication, they are now used to access a wide range of services. As their capabilities continue to widen, they will be increasingly used for tasks that were once only performed by computers. In advanced markets, voice calls and SMS are no longer the dominant services. This can especially be seen with smartphone users, who are increasingly making use of data-based services on a daily basis. [5].With the introduction of new, larger screen smartphones and tablets with all mobile-data-plan types, there is a continuing increase in usage in terms of gigabytes per month per user. The proportion of mobile users generating more than 2 gigabytes per month was 25 percent of users at the end of 2014 (Figure 2) [4].

The increasing popularity of smartphones in recent reports suggests that smartphone traffic has been growing 10 times faster than traffic generated from fixed networks [2]. The rising number of smartphone subscriptions is the main driver of mobile data traffic growth. Data traffic in the mobile phone segment is primarily generated by smartphones. There are large differences in data usage patterns between different networks, markets and user categories [5]. Smartphone speeds, generally $3 \mathrm{G}$ and higher, will be nearly three times higher than the overall average by 2019 . There is anecdotal evidence to support the idea that usage increases with the increase of the speed, although there is often a delay between the increase in speed and the increased usage, which can range from a few months to several years [4].

\section{Context of smart mobile devices usage}

People use their mobile devices in different ways, depending on their current situation. For instance, in an office situation people might be more likely to use their smartphones to make phone calls or check for next meetings, while at home devices might be used more to surf the Internet or watch movies [1]. The devices' strong integration into users' everyday lives gives a possibility to infer information also about the users' other, everyday habits [6]. 
Smartphones provide advanced functionality beyond voice and message services. Their widespread usage is advantageous for the realization of context-aware systems. Recent smartphones are equipped with a variety of sensors such as an accelerometer, gyroscope, GPS, microphone, image sensor, light sensor, proximity sensor, temperature and pressure sensors etc. These sensors can be an effective tool to understand the user's context which can lead to a development of context-aware applications [15].

Detecting significant places (meaningful to a user) and contexts has gained more and more interest also in the academia [16].

\subsection{Context of use}

The ISO 13407:1999 standard defines context as the characteristics of the users, tasks and the environment in which the system is used. This standard points out the need to know not just the users but also the contexts of use, because the context affects the usage situation similarly as the user and the technology in use [8].

The user context is any information that can be used to characterize the situation of the user. The information is extracted from data collected with the user's mobile devices by using a special purpose data collection platform. Information that can be used to describe the situation of the user includes a place derived from location data, time, and other people surrounding the user [17].

In this paper, the context of use represents a joint impact of smart mobile devices and mobile applications, used information and communications services and the user's environment.

Context information is seen as an important component in developing new, more personalized, mobile services and applications. The potential value of context information lies in its possibilities to predict presumable differences in user behavior and usage habits related to the different contexts/situations of the user. Context is any information that can be used to characterize the situation of an entity. An entity is a person, place, or object that is considered relevant to the interaction between a user and an application, including the user and applications themselves [16].

Understanding user context is a prerequisite for providing human-centered services that improve the quality of life. A smartphone is an appropriate device to infer user context because data on the frequent interactions between users and their devices can be easily collected using various kinds of embedded sensors. Furthermore, a smartphone makes it possible to generate and use social context, in addition to individual context, through Internet connectivity [7].

The design for different contexts of use should take into account not only the physical context (lighting, temperature, unstable usage positions, noise levels, etc.) and environmental factors (moving surroundings, network coverage, technology compatibility, etc.) but also the social context, i.e. people around the user and other, wirelessly connected people. In everyday life users typically adapt themselves to widely changing usage contexts [18].

Even though it is possible to discuss various context types of smartphone use and its users (social environment, activity, conditions, infrastructure, location etc.) location context has a great importance for tracking user's behavior. Accordingly, figure 3 shows a comparison of the use of smartphones based on different location context, in the USA and the UK. It is evident that the listed devices are mostly used at home, as a location context. Although there are some differences in the level of use in certain contexts, it is possible to conclude that the habits of the users from the observed country are very similar.

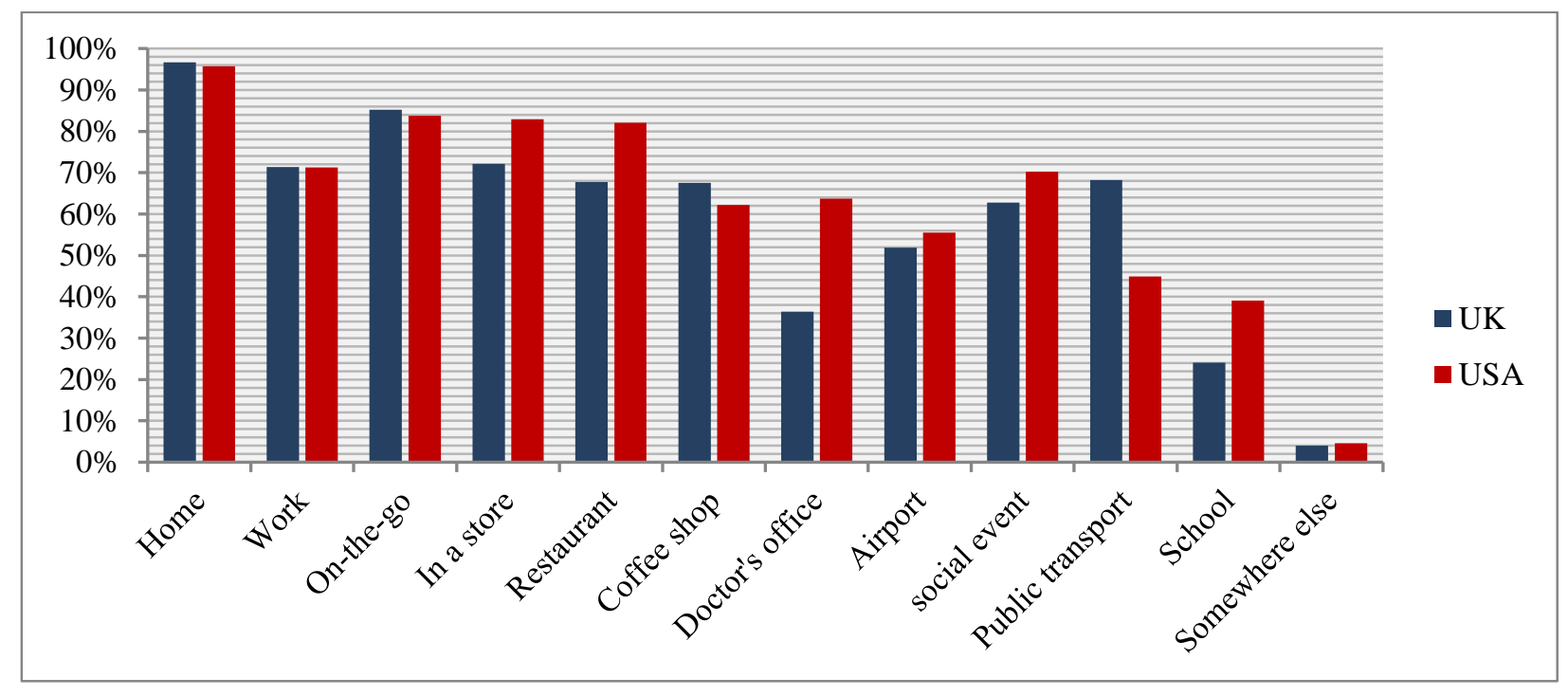

Fig. 3. Overview of location context of smartphone usage in the UK and the USA in 2013, [14] 
People spend more than 85 percent of their day indoors. In today's connected world, consumers expect to have instant access to the internet regardless of whether they are indoors or outdoors. Young people across 23 countries use their devices to access online content irrespective of location. In fact, this user segment accesses more online services when outdoors compared to the overall population. The most popular online activities performed by young people outdoors include watching TV/videos, gaming and online shopping [5].

Mobile end user context has been studied before with different methodologies, but inferring it from handsetbased data is still a relatively new approach [6].

\subsection{Smart mobile devices user behavior}

Smartphones enable users to access the Internet from anywhere at any time, empowering them to interact with brands in whatever context they choose [19]. The main usability challenges with mobile devices, applications and services are related to the widespread user population, the restrictions of the devices and technical infrastructures as well as the varying and demanding contexts of use. Mobile devices are not necessarily used while on the move; they can be used at home or at the office as well. However, the possibility to carry the device with the user introduces great variability to the context of use, which may even change in the middle of a usage session [8].

Analyzing characteristics of user interactions with their devices can benefit many research areas [1]. By combining the characteristics of smartphone usage sessions with context information, it is possible to examine the effect of end user context on the smartphone usage sessions. During a day, a user can be in quite different situations, such as commuting, concentrating on work at the office or relaxing at home. The different situations or contexts pose their own limitations and liberties to potential activities, including smartphone usage [16].

The programmability of smartphones gives a possibility to turn the devices into data collection platforms providing detailed information on user's smartphone usage habits. Smartphone usage sessions capture the user behavior and usage habits of a smartphone user on a high level. With the relatively comprehensive handset-based data which enables a production of time-wise linked context and session information, we can examine the effects of context on user behavior in the form of smartphone usage habits [16].

While smartphones will soon be ubiquitous, there is likely to be substantial variation in how different age groups use them. Although over-55s are buying smartphones, getting them to fully exploit the data functions is a key opportunity for network operators [20].

The availability of context-aware smartphone services makes several scenarios possible. For example, using social-context information, a company scouting for locations to display its advertisements can obtain useful information on various places frequented by urban women in their twenties in the evening. Parents could acquire information on where their children usually go at certain times of the day. Furthermore, a smartphone makes it possible to generate and use social context, in addition to individual context, through Internet connectivity [7].

Smartphones offer a platform for new kinds of applications and services and the devices are more and more integrated into the user's everyday lives. The capabilities of the smartphones give a possibility to collect usage data by using on-device data collection software. The data in turn can be used to infer information about the smartphone usage habits and user behavior [17].

\section{Previous researches}

A plethora of studies focus on understanding smartphone usage and user's behaviors from different perspectives have previously been studied.

To improve understanding on user's behavior and mobile service usage, in the study [3] traditional survey-based data collection was complemented with a range of automated measurement methods. In that study, the alternative data collection methods are analyzed based on a literature study and practical experiences. Authors in [21] used a robust data collection tool which allows reliable collection of information on Android smartphone usage. Their analysis of the dataset demonstrates considerable diversity in behavior between users but also over time. They further demonstrated the value of handset-centric data collection by presenting case-study analysis of human mobility, interaction patterns, and energy management and identified notable differences between given results and those found by other studies.

A study [22] attempted to address the lack of sufficient knowledge about how, where, and when mobile applications are used at a national scale. It focused on profiling the usage patterns of mobile apps and investigate how, where, and when smartphone applications are used from spatial, temporal, and user perspectives. Research [23] analyzed the mobile data usage of different user groups in different geographical regions and different time frames. Authors have observed that there are interesting daily trends in the data usage. Moreover, some specific events and holidays have a significant impact on the data usage.

Research [24] investigated the current dynamics of the Malaysian market for smartphone and the usage behaviors of consumers. Furthermore, consumers' usage behaviors such as using a smartphone for email, web browsing, gaming, and document reading were examined. A study [25] defined a handset-based method for analyzing mobile service usage. 
The research method can be used in conducting empirical studies of consumer behavior. It combines collected handsetbased usage data with survey data, both of which are important in modeling consumer behavior.

This study is complementary to the studies mentioned above and, additionally, gives a presentation of the conceptual model which allows identification of various groups of smartphone users and their behavior. At the same time, the conceptual model shows the possibility of user's segmentation depending on their behavior within the different daily situations (contexts).

Smartphones are equipped with a variety of sensors such as an accelerometer, gyroscope, GPS, microphone, image sensor, light sensor, proximity sensor, temperature and pressure sensors etc. These sensors can be an effective tool to understand the user's context which can lead to the development of context-aware applications. A group of studies attempted to investigate smartphone usage in different usage context [1], [2], [6], [16].

Research [1] studied mobile device usage characteristics like session length, interaction frequency, and daily usage in the locked and unlocked state with respect to location context. Authors in [6] and [16] studied the relation between mobile device usage and end-user context using 140 smartphones. They found that usage sessions are longer in home context while more frequent in the office context. According to their usage session analysis, smartphone usage is highly diversified across users. The results imply that mobile services and applications need to adapt to user behavior in order to be personalized enough, and that context awareness can indeed be a worthwhile step towards this.

In research [2] author analyzed to understand the workload characteristics of smartphone traffic and study the relationship between participant contexts and smartphone usage. The collected dataset suggests that even in a small group of participants a variety of very different smartphone usage patterns occur. Dependencies between smartphone usage and context (where they are, who they are with, at what time, and over which physical interface) are investigated in their work.

Authors believe that this study makes an important step in addressing the lack of knowledge of smartphone usage behaviors using monitoring system. Overall, this study is the first that attempts to address the lack of sufficient knowledge about the model which would allow to identify and predict the user behavior and habits using smart mobile devices and connected measurement system.

\section{Conceptual model of context identification and prediction of user behavior}

Smart mobile devices can be used to gather information that can characterize the situation of the user. In this research, it is assumed that the smart mobile device has only one user and that the user has the device always with him. With these assumptions, it can be presumed that the information extracted from the smart mobile device can be used to characterize the situation of the user.

The integration of smart mobile devices into user's everyday life is the background for the development of the conceptual model that will make conclusions about their habits and patterns of their behavior based on the collected data. The data analysis can determine user's affiliation to a particular segment of users.

For the purposes of this research, the figure 4 shows the conceptual model - a technical solution for the collection and analysis of data and subsequent analysis of results (reports). The model consists of three main segments:

- Data collection

- Analysis of the collected data

- Generating the reports

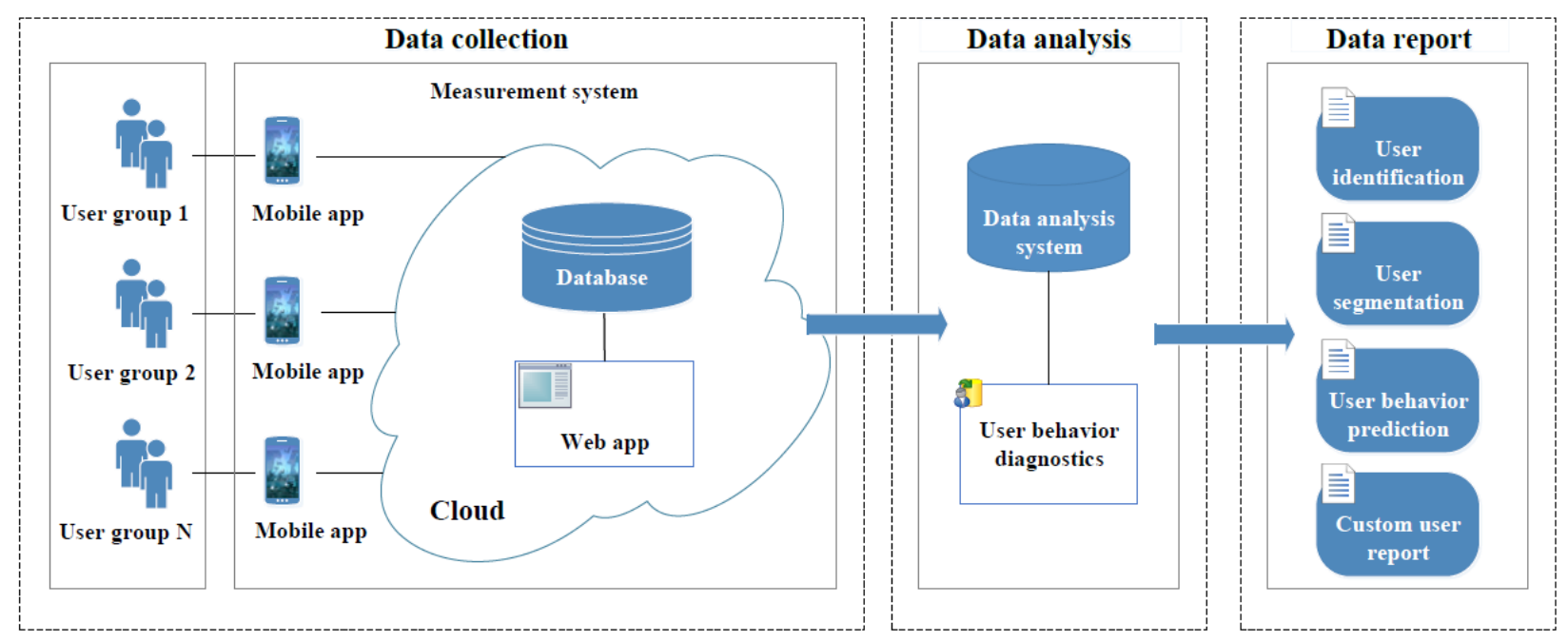

Fig. 4. Conceptual model for identification and prediction of user behavior using smart mobile devices 
The data collection process is conducted using smartphone devices used by their owners (i.e. users). The installed application is part of the measurement system within the smartphone device which performs customer data collection. The measurement system also includes a collected database about user's behavior and web application for the display of the collected data. The specified database and web application are parts of the cloud service.

Deployment of a monitoring system sharply reduces the time of availability and reduces damages which can be consequently caused [26]. The segment of data analysis by the function of collected data analysis allows the identification of the users and their behavior as well as segmentation of the users having similar behavioral characteristics.

The third segment of the conceptual model represents a part with the purpose of generating the reports based on the analysis of the collected data. Within the listed segment, it is possible to generate previously defined reports (user identification, segmentation, and behavior prediction) or to say, custom reports based on current needs (custom data report).

Smartphones and similarly "always on, always connected" devices allow access to a wealth of information [27]. The functionality of the conceptual model includes the collection of the data about the smartphone user's behavior in different contexts and the frames of time. The listed concept can be implemented in defined groups of users: students, pensioners, the blind and visually impaired users and the users of specific applications (such as services sharing transportation, carpooling). The data analysis will determine the affiliation to a particular user segment - a group of users of common characteristics in the use of devices, ICT services, and applications.

Nowadays, smartphones play an ubiquitous role in accessing and processing information, most of them having a myriad of integrated sensors that makes them capable of generating information with high accuracy and precision [28]. Collecting usage information on smartphones is difficult: The collection mechanism itself needs to be built and deployed to a diverse group of participants running a multitude of devices in the wild; data must be collected for extended periods of time to overcome novelty effects and find long-term trends. Consequently, researchers are often forced to rely on their intuition or on the results from limited small-scale studies [21].

\section{Conclusion}

Smart mobile devices can be a part of a data collection platform which provides detailed information on smartphone user's habits. Smartphone's sensors can be an effective tool to understand the user's context, behavior and habits. The context of use of smart mobile devices represents a joint impact of smart mobile devices and mobile applications, used information and communications services and the user's environment.

Results of a new technical solution offered by the conceptual model presents a complimentary upgrade of existing researches. The stated platform provides the architecture of technical solution for the collection of data about users and their subsequent analysis. The implementation of individual segments of the model depends on the used platforms and it is presented as a concept within the model.

Limitations of the research and the author's aproach are related to the material and financial possibilities of procurement of platform for monitoring the smartphones and the users. The mentioned platform will enable the collection and analysis of user's data, including further and described objectives which is the answer to the research question.

Further research is going to be an upgrade of existing conceptual model which makes the basis of solution for collecting data about users. Users will be segmented into specific categories based on different preferences and behavior. The research will observe specific groups of users, such as students, people with reduced mobility, the blind and visually impaired, users of car-sharing service etc. The data about the habits of specific groups of users will be collected, and the obtained database of information will be used for subsequent data analysis. Described process will allow the identification of new customers and prediction of their future behavior on the basis of previous findings.

\section{Acknowledgements}

This research has been carried out as part of the project "Research of the context of the use of smart mobile devices and related information and communication services", funded as part of the program "Short-term Financial Support of Research", University of Zagreb, 2015.

\section{References}

[1] D. Hintze, S. Scholz, and R. D. Findling, Mobile Device Usage: Characteristics by Usage Type, Context and Form Factor, Proceedings of the 12th International Conference on Advances in Mobile Computing and Multimedia, 2014, pp. $105-114$.

[2] S. Kaisar, Smartphone Traffic Characteristics and Context Dependencies, University of Saskatchewan, 2012.

[3] A. Kivi, N. L. P. O. Box, Measuring Mobile User Behavior and Service Usage: Methods, Measurement Points, and Future Outlook, Methods, 2005, pp. 1-15.

[4] Cisco Systems Inc., Global Mobile Data Traffic Forecast Update, Cisco Visual Networking Indeks, 2010 - 2015, 2011. 
[5] Ericsson Mobility Report, Mobility Report On The Pulse of the Networked Society, 2014.

[6] T. Soikkeli, J. Karikoski, H. Hämmäinen, Characterizing Smartphone Usage, International Journal of Handheld Computing Research, 2013, vol. 4, no. 1, pp. 15-36.

[7] Y. Chon, H. Cha, A smartphone - Based Context Provider for Location-Based Services, Pervasive Computing, vol. 11, pp. 58-67, 2011.

[8] E. Kaasinen, User Acceptance of Mobile Services - Value, Ease of Use, Trust and Ease of Adoption, 2005.

[9] D. Peraković, S. Husnjak, V. Remenar, Research of Security Threats in the Use of Modern Terminal Devices, 23td International DAAAM Symposium, 2012, vol. 23, no. 1, pp. 545-548.

[10] S. Husnjak, D. Perakovic, I. Jovovic, Possibilities of using speech recognition systems of smart terminal devices in traffic environment, Procedia Engineering, vol. 69, pp. 778-787, 2014.

[11] Nielsen, A. Global Snapshot, The Mobile Consumer, February 2013, pp. 1-11.

[12] Ericsson Traffic Exploration Tool, Global Smartphone Subscriptions, 2010 - 2017, $2015 . \quad / /$ http://www.ericsson.com/TET/trafficView/loadBasicEditor.ericsson.

[13] Mobidia \& Ovum Ltd., Smartphone \& Tablet Usage Trends \& Insights 4G LTE and Wi-Fi Powering Data Consumption, 2015.

[14] Google Inc., Our Mobile Planet - smartphone usage in 2013

[15] Q. U. Ahmed, Activity Recognition Using Smartphone Accelerometer and Gyroscope Sensors Supporting ContextBased Reminder Systems, Context Aware Reminder System, Faculty of Computing at Blekinge Institute of Technology, 2014.

[16] T. Soikkeli, J. Karikoski, H. Hämmäinen, Diversity and End User Context in Smartphone Usage Sessions, Next Generation Mobile Applications, Services and Technologies (NGMAST), 5th International Conference, 2011, pp. $7-12$.

[17] T. Soikkeli, The Effect of Context on Smartphone Usage Sessions, Aalto University School of Science, 2011.

[18] E. Kaasinen, V. Roto, K. Roloff, T. Vainio, W. Maehr, D. Joshi, S. Shrestha, User Experience of Mobile internet Analysis and Recommendations, Mob. HCI, Special issue Mobile Internet User Experience, vol. 1, no. 4, 2009.

[19] F. Consulting, The State Of Mobile Apps For Retailers, 2015.

[20] Deloitte, Deloitte Media Consumer Survey 2014, 2014.

[21] D. T. Wagner, A. Rice, A. R. Beresford, Device Analyzer: Understanding Smartphone Usage, International Conference on Mobile and Ubiquitous Systems: Computing, Networking and Services, 2013, pp. 1-12.

[22] Q. Xu, Z. M. Mao, A. Arbor, J. Erman, F. Park, A. Gerber, J. Pang, S. Venkataraman, Identifying Diverse Usage Behaviors of Smartphone Apps, Proceedings of the 2011 ACM SIGCOMM conference, Internet measurement conference, 2011, pp. 329-344.

[23] E. Bulut, S. Troy, Understanding User Behavior via Mobile Data Analysis, 2015, pp. 1548-1553.

[24] M. A. Osman, A. Z. Talib, Z. A. Sanusi, A Study of the Trend of Smartphone and its Usage Behavior in Malaysia, 2012, vol. 2, no. 1, pp. 275-286.

[25] H. T. Verkasalo, Handset-based Analysis of Mobile Service Usage, Helsinki University of Technology, Faculty of Electronics, Communications and Automation, Department of Communications and Networking, Doctoral dissertation, 2009.

[26] M. Matýsek, M. Adámek, M. Mihok, Monitoring of active devices in computer networks, 25th DAAAM International Symposium on Intelligent Manufacturing and Automation, DAAAM 2014, vol. 25, no. 1, pp. 521-530

[27] G. Maggiore, C. Santos, A. Plaat, Smarter smartphones: understanding and predicting user habits from GPS sensor data, Procedia Computer Science, 2014, vol. 34, pp. 297-304

[28] F. Duarte, A. Lourenco, A. Abrantes, Classification of Physical Activities using a Smartphone: evaluation study using multiple users, Procedia Technology, 2014, vol. 17, pp. 239-247 